\title{
O.S.P.
}

L'orientation scolaire et professionnelle

$45 / 3$ | 2016

Varia

\section{Francis Bacon et le Life design}

Francis Bacon and the life design

Jacques Pouyaud

\section{OpenEdition}

Journals

Édition électronique

URL : http://journals.openedition.org/osp/5017

DOI : 10.4000/osp.5017

ISSN : 2104-3795

Éditeur

Institut national d'étude du travail et d'orientation professionnelle (INETOP)

Édition imprimée

Date de publication : 15 septembre 2016

ISSN : 0249-6739

Référence électronique

Jacques Pouyaud, « Francis Bacon et le Life design », L'orientation scolaire et professionnelle [En ligne], 45/3 | 2016, mis en ligne le 01 septembre 2018, consulté le 16 décembre 2020. URL : http://

journals.openedition.org/osp/5017; DOI : https://doi.org/10.4000/osp.5017

Ce document a été généré automatiquement le 16 décembre 2020.

(c) Tous droits réservés 


\title{
Francis Bacon et le Life design
}

\author{
Francis Bacon and the life design
}

Jacques Pouyaud

\section{Introduction}

1 Francis Bacon (1909-1992) est incontestablement l'un des plus grands peintres du $\mathrm{Xx}^{\mathrm{e}}$ siècle. Il occupe, selon Kundera (1996), la place ultime d'un des « derniers peintres dont le langage est encore huile et pinceau » (p.15), de ceux qui, comme Beckett en littérature, n'ouvrent pas le chemin «mais le referment»(Kundera, 1996, p. 15). Dernier grand peintre "classique", Bacon est aussi resté unique en son genre. Autodidacte, rejeté dès ses débuts du mouvement surréaliste dans lequel il pensait initialement s'inscrire, il se tiendra ensuite prudemment à l'écart de tous modes et courants. Son expérience de peintre a été celle d'un solitaire (Bacon, 2012), produisant une œuvre au singulier, tout en restant en phase directe avec les enjeux de la modernité.

2 Dans le siècle de la subjectivité (Guichard, 2000), durant lequel «les gens [...] ne peuvent pas vraiment imaginer une humanité sans guerre » (Bacon, 2012, p. 123), et où «le moi fatalement se dérobe » (Kundera, 1996, p. 10), Bacon, plus qu'aucun autre, met en scène la figure humaine en offrant un miroir à l'homme postmoderne dans sa chair tragique, sa vie futile, absurde, et sa confrontation à l'horreur et à la violence.

Dans l'histoire de l'art, Bacon est peut-être celui qui articule art primitif et art moderne. Il est primitif par son attachement instinctif au geste et au corps dans une matérialité crue. Il est moderne dans sa tentative d'accès au sujet par la voie de cet instinct matériel. Chez Bacon, «la main du peintre [...] se pose d'un geste brutal sur un corps, sur un visage, dans l'espoir de trouver, en lui et derrière lui, quelque chose qui s'y est caché » (Kundera, 1996, p. 10).

4 Dans le champ des sciences humaines, la fin du $\mathrm{xx}^{\mathrm{e}}$ siècle a également vu se développer une vision postmoderne de l'individu. Parmi les constats fréquemment abordés ; celui de l'effritement des relations sociales devenues plus fluides et changeantes, modelant 
ainsi une "société liquide » (Bauman, 2007); celui de l'incertitude devenue une norme sociale (Ehrenberg, 1995); celui du sujet devenu multiple face aux développements des nouvelles technologies et des réseaux avec le risque de «saturation» (Gergen, 2009); celui de la désynchronisation (Rosa, 2012) qui donne à notre quête de sens le parfum d'un risque, celui de l'aliénation, quand le sujet est « pris dans des activités à ce point différentes qu'il n'est plus possible [...] de les accorder entre elles " (Leblanc, 2012, p. 187) ; celui des risques enfin d'une injonction sociale de mobilisation infinie de soimême (Sloterdijk, 2000). Tous ces constats dessinent la figure d'un sujet de plus en plus privé de sa relation au monde réel, un individu postmoderne à la fois mobile, mobilisable et mobilisé, dont le surfeur ou le skateur représente l'archétype fluide (Fischbach, 2011).

5 Dans cette rapide analyse des enjeux postmodernes du sujet, le mouvement, l'attachement à la réalité et au corps, la variation de soi et le risque inhérent de la perte de soi sont au centre d'une tentative plus globale de compréhension de ce que l'on pourrait appeler la "construction de soi ", aujourd'hui au cœur des parcours et des problématiques d'orientation. C'est ce projet du soi en mouvement que nous voulons aborder dans une analyse à la fois artistique et scientifique du travail de Bacon. L'entreprise qui consiste à relier une œuvre artistique et des modèles psychologiques pour en tirer une «composition heuristique » peut sembler hasardeuse. Elle pourrait aussi paraître incongrue pour ce peintre qui « désavoue la critique littéraire et conçoit sa peinture comme directement expressive " (Harrisson \& Wood, 1997, p. 688). C'est peut-être pourtant cette dernière caractéristique qui permet le rapprochement de Bacon avec le psychologue. Et pour comprendre comment son œuvre parle du sujet postmoderne et de la construction de soi, il faudra partir du processus créatif luimême.

6 Bacon dans ses peintures nous parle « visiblement ", de manière « sensationnelle » du sujet, parce qu'il utilise un procédé créatif dans lequel sa propre sensation est mise en forme sur la toile de manière directe. Bacon décrit par exemple son travail sur sa première version de Peinture de 1946 :

«Je voulais faire un champ avec un oiseau qui le survole et j'avais placé tout un tas de repères sur la toile pour cela, et puis d'un coup, les formes que l'on voit sur cette toile ont commencé d'apparaître, elles se sont imposées à moi. Ce n'était pas ce que je comptais faire, loin de là. C'est arrivé comme cela et j'étais plutôt étonné de cette apparition. [...] C'est arrivé de façon inattendue, comme un accident. Il était prévu quelque chose, et puis, d'une façon tout à fait étonnante, quelque chose d'autre est arrivé. C'est à la fois accidentel et en même temps complètement évident » (Bacon, 2012, pp. 64-65).

7 À travers ce procédé créatif, Bacon cherche à faire une peinture qui ne parle pas à la raison, mais directement au système nerveux. C'est " une tentative pour que la figuration atteigne le système nerveux de manière plus violente et plus poignante " (Sylvester, 2013, p. 19). Le hasard sert à préserver l'expression directe de soi. Mais, comme le souligne Deleuze (2002), ce hasard « ne désigne plus du tout des probabilités, il désigne maintenant un type de choix ou d'action sans probabilité [...] le hasard manipulé » (p. 89), qui devient alors une méthode créative.

Bacon décrit cette méthode ainsi :

«tandis que l'on travaille dans une certaine direction, on essaye d'aller plus loin dans cette direction, et c'est alors qu'on détruit l'image que l'on avait faite et que l'on ne retrouvera plus jamais. C'est alors aussi que surgit quelque chose qu'on 
n'attendait pas et qui arrive inopinément. [...]. Le plus étonnant, c'est que ce quelque chose qui est apparu comme malgré soi est parfois meilleur que ce que l'on était en train de faire " (Bacon, 2012, pp. 66-67), mais, "cela n'a rien à voir avec l'inspiration [...] non, c'est quelque chose qui provient du travail lui-même et qui surgit à l'improviste.» (Bacon, 2012, p. 71) «Ce qui finalement apparaît dans le meilleur des cas sur la toile, c'est probablement un mélange entre ce qui est voulu par le peintre et ces accidents. » (Bacon, 2012, p. 68)

Si tout ce processus se déroule dans le «brouillard» (tout comme la situation de choix professionnel qui est souvent incertaine, inquiétante, et sans visibilité), il s'effectue néanmoins, non pas au hasard, mais à partir d'un travail et d'une certaine discipline. Bacon nous dit, "c'est le mélange de cet entraînement et de ce qui vient sans qu'on l'attende qui fait, je crois, ma peinture » (Bacon, 2012, p. 72). Cette dernière est alors loin d'un simple procédé de reproduction ou d'illustration comme peut l'être la photographie, dont Bacon soulignait qu'elle "n'a rien à voir avec l'aventure que représente le travail sur la toile » (Bacon, 2012, p. 92). Le travail du peintre sur la toile, dont les tableaux de Bacon sont les traces, est une aventure créative, développementale, de quelqu'un qui cherche à « parvenir à faire quelque chose dans sa vie qui lui donne un sens" (Bacon, 2012, p. 93). La manière dont Bacon décrit son processus de création nous montre à quel point ce dernier est proche de la question de la construction de soi.

10 Parce que l'orientation parle aussi de la manière dont les personnes peuvent se construire dans leur environnement social et professionnel, elle est un champ de recherche propice à la modélisation de la construction de soi. Au tournant des années deux mille ont ainsi émergé plusieurs approches en psychologie de l'orientation. Souvent construites sur des perspectives narratives, elles mettent au centre de la pratique de conseil le projet d'accompagnement à la construction de soi et la notion d'identité narrative (e.g. Hartung, 2013; McMahon \& Patton, 2005; McMahon \& Watson, 2011). Conjointement à ce mouvement, Savickas et al. ont introduit le terme de "Life design" pour nommer un paradigme dans lequel pouvait se concevoir une psychologie du conseil renouvelée dans les problématiques sociétales et individuelles des sociétés postmodernes (Savickas et al., 2010). L'œuvre de Francis Bacon constitue, nous semble-t-il, un corpus particulièrement saillant pour comprendre ce nouveau paradigme, dont il fournit une illustration tant sur le plan pratique que conceptuel. Après être revenu sur notre méthode d'analyse, le paradigme du Life design sera présenté à partir d'une métaphore de l'identité comme une bicyclette, qui servira ensuite de cadre d'analyse de l'œuvre de Bacon comme une trace artistique de sa propre construction.

\section{Soi comme sujet principal : méthode d'analyse}

11 Le sujet principal de Bacon est la Figure. Dans sa solitude créative, il s'est souvent représenté lui-même. Peintre de l'autoportrait, l'œuvre de Bacon est une mise en forme de sa propre subjectivité en action. Analyser les œuvres de Bacon les unes par rapport aux autres, c'est analyser cette subjectivité dont chaque peinture est un témoin, un enregistrement momentané de sa propre construction. L'œuvre entière, dans sa répétition de portraits raconte l'aventure du recollage de soi à l'échelle d'une vie.

12 L'œuvre d'art conçue sous cet angle rappelle les travaux de Meyerson et de la psychologie historique (Meyerson, 1995). Pour Meyerson, « il existe des produits de la 
pensée qui témoignent, bien mieux que les artefacts de la psychologie empirique, des conditions de fonctionnement de l'esprit humain" (Fruteau de Laclos, 2007, p. 121). Cette idée s'appuie sur une fonction essentielle de l'esprit qui est la fonction d'objectivation comme "capacité à produire des formes objectives signifiantes " (Fruteau de Laclos, 2007, p. 122). En ce sens, il n'y a pas de pensée autre que dans cette activité d'objectivation productrice de traces. Les traces de l'activité de pensée, ou " œuvres ", une fois réalisées deviennent des produits indépendants de la pensée qui les a hébergée, et, de fait, des supports d'analyse pour le psychologue. Ils se détachent de leurs conditions de production pour devenir des produits sociaux, culturels, et des outils psychologiques ${ }^{1}$ (Vygotski, 1997), intégrés à la pensée historique des hommes. La difficulté méthodologique pour le psychologue qui veut comprendre le sujet à partir de ses œuvres vient alors du fait que ces dernières, une fois émancipées de leur créateur, ne peuvent s'analyser pleinement que dans le rapport qu'elles entretiennent avec l'activité de pensée qui les vu naître. La connaissance des conditions de l'activité de création, au-delà des traces restantes est alors essentielle.

Chez Bacon, ce problème méthodologique se résout de deux manières : les conditions de création sont accessibles dans les entretiens réalisés par Bacon (principalement avec Leiris, Archimbaud et Sylvester). Dans ces entretiens, Bacon se livre à un ami comme un miroir de lui-même. Mais la création apparaît aussi sur la toile elle-même et dans l'analyse que l'on peut faire de la confrontation des peintures entre elles. Nombre d'œuvres de Bacon ne sont que des répétitions du même sujet (souvent lui-même), figées après un processus de création singulier. Elles sont des variations de soi construites sur des leitmotivs qui constituent un moyen d'accès indirect aux processus de création. Chez Bacon, la méthode créative consiste à travailler le hasard pour que les peintures soient les moins signifiantes possible. Les peintures de Bacon ne sont pas $a$ priori des œuvres qui sont faites pour dire, mais pour « être » : " elles sont seulement ». Le processus d'objectivation chez Bacon est comme décapité, coupé de la réflexion. Pour Bacon, sujet de lui-même, les peintures jouent le rôle d'instruments psychologiques au sens de Vygotski. L'instrument psychologique ne vise pas le travail sur les objets comme le fait un outil classique, mais tend à exercer une influence sur le psychisme propre. C'est un outil tourné vers soi-même. "Dans l'acte instrumental, l'homme se contrôle lui-même de l'extérieur, à l'aide des instruments psychologiques " (Vygotski, 1985, pp. 41-43). Les peintures de Bacon s'analysent alors comme des modes de régulation de soi.

Dans cette perspective et en lien avec Meyerson, l'identité s'apparente à un système adaptatif de résolution de conflit dans l'activité. Elle produit une «œuvre de soi » au sens d'une objectivation des sensations internes issues des expériences d'altérité transformées en formes narratives et historiques. Suivant cette logique, les tableaux sont comme des enregistrements d'une identité en mouvement, coupés de significations, mais dont il serait possible de reconstituer le parcours narratif par une juxtaposition historique. L'analyse de ce parcours dépasse largement le cadre de cet article et devrait combiner pour se faire les points de vue d'historiens de l'art, de critiques, de psychologues, de philosophes, etc. Notre analyse se bornera à illustrer comment un modèle de la construction de soi peut se lire dans la trace artistique laissée par ce grand peintre. 


\section{La métaphore de l'identité comme un vélo (bicycle)}

15 Le paradigme du Life design place au centre de sa proposition l'idée que l'identité est une méta-ressource d'adaptation face aux variations de l'environnement et aux événements de l'existence. C'est à travers l'identité narrative que le sujet se construit de manière active sur la base de la quête perpétuelle d'une « demeure " pour soi dont le caractère "accueillant» va participer de sa stabilisation dans une histoire construite. Pouyaud (2014) propose, pour comprendre et modéliser le rôle de l'identité narrative dans la construction de soi d'utiliser la métaphore du vélo (bicycle - Be-cycle).

L'idée générale de cette métaphore est que, comme le vélo, l'identité est un outil qui permet de "se conduire» dans la vie. Les trajectoires de vie sont les chemins empruntés, et le vélo un moyen de l'action quotidienne permettant d'avancer, de s'adapter, de choisir et de s'engager dans une direction à la recherche d'une « demeure accueillante ». Il s'agira de se conduire dans un contexte, au milieu d'une circulation, sur des voies rapides ou des chemins encaissés, parfois pour aller quelque part, ou juste pour déambuler, sur des chemins habituels ou inconnus. L'identité, comme le vélo, est un instrument de la construction de soi dans le sens où elle est à la fois l'armature du mouvement de soi et le moyen du trajet.

17 Tout comme le mouvement donne l'équilibre au vélo, l'identité tient le sujet en équilibre par un effort qui l'oblige à avancer. Le paradoxe identitaire, impossible projet qui consiste à faire coexister mêmeté et ipséité en constitue le moteur. Le mouvement est par essence au cœur de l'identité, mais il n'est pas pour autant un mouvement perpétuel. Pour jouer son rôle de véhicule, l'identité a besoin des efforts de l'individu qui maintient le rythme et dirige le parcours. L'effort à fournir dans le trajet peut être très important lors de transitions ou traumatismes à affronter (à l'image d'une côte à grimper), ou minimum dans le cas de périodes de vie plus stabilisées.

18 La capacité du vélo à nous mouvoir tient ainsi d'un dosage subtil fait par le cycliste entre les caractéristiques du terrain et les efforts de pédalages jugés nécessaires par les conditions de ce terrain (des capacités adaptatives). Les déséquilibres et encaissements du terrain imposent des stratégies de conduites (des stratégies identitaires) de maintien, d'anticipation, ou de dépassement de soi dans l'activité, qui dépendent des ressources propres au sujet (capital identitaire).

19 La notion d'identité est aussi liée à la temporalité. Elle se comprend dans cette métaphore comme un recyclage (un bi-cycle). Le dialogue identitaire ne répète pas, en effet, le sujet, mais le reconstruit, le renouvelle, le recycle dans des variations narratives. Ce processus de renouvellement est tourné vers l'avenir et conçu pour " aller vers » (orienté vers un but). Le seul recul possible sur un vélo est le retour vers un point de départ sans marche arrière. Ce retour est en fait une avancée, un parcours, un développement qui donne à la course identitaire l'allure d'une avancée en spirale reliant de manière dynamique le passé et le futur.

Les parcours d'orientation sont faits d'adaptation aux terrains et aux chemins que l'on emprunte, et l'identité se construit par adaptation réciproque avec les contextes de vie. Tous les vélos se ressemblent et fonctionnent sur le même principe. Les changements sont rarement radicaux, mais chacun se construit et s'use différemment selon les terrains rencontrés et les améliorations créatives trouvées en cours de route face à l'adversité du terrain. Les aménagements nés de ces conflits deviennent des ressources 
identitaires, des capacités adaptatives. Les valeurs et les intérêts construits et développés au cours de l'expérience en sont des exemples. Ce sont ces produits du mouvement du soi, ces traces de l'activité de construction nées des variations constitutives et progressives du soi lors des décalages vécus, des déséquilibres, qui modèlent (design) notre existence.

Dans cette métaphore, plusieurs modèles théoriques liés aux paradigmes constructivistes peuvent être mobilisés (voir figure 1).

La notion d'identité narrative développée par Ricœur $(1988,1992)$ en est le cadre général. Ricœur conçoit l'identité comme un rapport, un mouvement entre deux pôles (les deux roues du vélo) constitués d'un côté par l'idem (le sentiment d'être le même), et de l'autre l'ipse (le sentiment d'unicité). L'identité est la colle narrative qui fait tenir ces deux pôles ensemble dans une logique temporelle. L'identité narrative n'est donc pas stable, mais au contraire volatile, réinterprétée au travers des événements de vie, remodelée au fil des narrations sur soi. Pour Ricœur, cette construction est dynamisée par une triple expérience de la différence : un rapport à la chair, à autrui et à soi-même (sa propre conscience). Ce triptyque de l'altérité serait alors le "cadre» d'un déploiement de l'identité (le cadre ou « l'armature » du vélo), sa structure dynamique, ce qui en donne la robustesse.

Parce que ces expériences d'altérité sont des supports de narration sur soi, elles prennent sens dans une dynamique de dialogue qui nécessite de définir les adresses et les places discursives qui le composent. Pour illustrer cet aspect nous faisons un parallèle entre la dynamique dialogique en jeu dans la construction de soi et les échanges numériques dont les adresses mail sont les supports. Une adresse mail (X@Y) est composée de deux dimensions « $\mathrm{X}$ » et « $\mathrm{Y}$ » mises en relation " @ " pour définir une identité virtuelle, c'est-à-dire une potentialité de dialogue. Autrement dit, l'adresse mail exprime dans sa première partie un représentant (de soi, d'autrui - X), qui est un rapport d'altérité, et dans sa deuxième partie un lieu d'hébergement de ce représentant (de soi et d'autrui - Y), qui est le positionnement de ce rapport. Les deux éléments mis en relation (@) construisent un représentant de soi (une identité numérique X@Y) qui définit ainsi une adresse et sert de support au dialogue. La multiplicité des adresses pouvant être créées renvoie aux identités multiples du sujet postmoderne, morcelé dans une variété de contextes de vie. Les trois expériences dynamisantes de l'identité décrites par Ricœur sont notées selon cette illustration comme des " adresses de soi " (au sens donc d'adresses mail: moyen d'hébergement, de communication, et d'identification) : «être@ »soi / autrui / conscience, rapports d'altérité hébergés chez soi comme un corps, un autrui ou une conscience (de soi-même comme un autre). 
Figure 1. Métaphore de la construction de soi

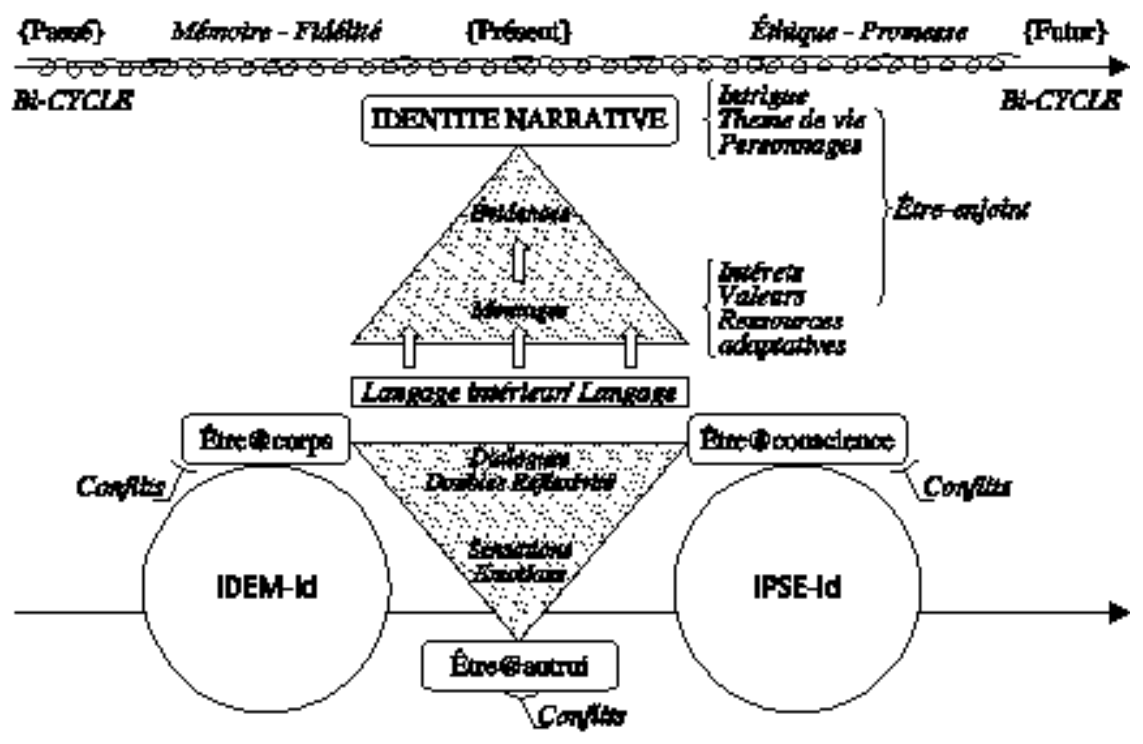

Figure 1. The metaphor of self construction Les adresses qui servent de relais aux dialogues peuvent être rapprochées des
propositions de Guichard et de son modèle "se faire soi » $(2004,2009)$. S'appuyant sur les propositions de Jacques (1982), la construction de soi est décrite comme un
processus dialogique d'interprétations permanentes de soi, basées sur les écarts perçus à partir de trois positions dialogiques : Je, Tu et Il, que nous rapprochons des adresses issues des niveaux d'expérience d'altérité décrits plus haut. Être@corps est l'adresse de laquelle s'exprime le niveau pragmatique de la confrontation au réel. Elle n'est pas forcément première, mais elle sert d'assise, d'ancrage physique à l'identité (la selle du bicycle) et peut se rapprocher d'un dialogue dans la position du «Je ». Ettre@conscience est l'adresse du niveau le plus désincarné et le plus cognitif. C'est celle qui offre une direction à l'identité (le guidon du bicycle). Elle vise la conduite vers une " bonne vie ", et se rapporte à un dialogue de soi-même comme un autre, un « Il ». Enfin, Être@autrui est peut-être l'adresse de l'expérience d'altérité la plus dynamisante (les pédales du vélo). C'est le point de vue des autres sur soi-même ou de soi-même vu " de l'autre », comme un « Tu ». Selon Guichard, durant les dialogues de conseil en orientation notamment, s'opère une 
d'une identification à une personne ou un système de représentations stabilisé. Ces processus qui visent à se "penser comme " conduisent à des certitudes sur soi, des évidences, à propos de qui je suis et veux être. Leur but est de certifier le sujet dans un parcours, et de l'inscrire dans une continuité. Dans le jeu continué de la réflexivité, on pourrait voir cette seconde forme comme le travail de consolidation des interprétants évoqués plus haut. Quand les «je » et les «ils » s'interchangent et se rapprochent dans les tours de paroles, alors l'adresse de soi tend à se stabiliser dans une forme de certitude à vouloir "être comme». Au milieu du triptyque constituant le cadre du bicycle, les deux types de réflexivités décrites par Guichard seraient ainsi à l'œuvre, tantôt pour assurer la course, tantôt pour la renouveler.

Concernant la nature des dialogues, l'hypothèse suivante est proposée : le processus de double réflexivité se transforme en actes de parole à travers l'utilisation conjointe du langage inter- et intra-individuel. Cette proposition s'appuie sur les travaux de Vygotski sur le rôle du langage et du langage intérieur comme outil psychologique (Vygotski, 1997). Les deux formes de langage (intra / inter) peuvent être considérées comme des moyens de "montage » (au sens cinématographique) des expériences du sujet. Elles donnent sens aux expériences et découlent en partie des émotions vécues dans l'action (Guichard \& Pouyaud, 2014). Le montage pourrait se traduire sur un premier niveau identitaire par la construction de marqueurs "visibles », et observables des individus; sentiments, attitudes, intérêts, valeurs, ressources adaptatives, compétences, etc., nommés plus haut de manière générique comme des certitudes ou des « évidences de soi ». Young et Valach $(2004,2006)$ décrivent dans leur théorie de l'action dirigée en contexte comment l'identité narrative s'enracine dans l'action et l'émotion. Les auteurs expliquent comment le niveau pragmatique de l'action est remodelé au travers des dialogues pour être transformé en trajectoire à long terme, que les auteurs décrivent comme des "carrières". À un niveau supérieur de ce dispositif adaptatif dynamique, le montage aboutit à une organisation, une synthèse identitaire des traces sensitives prenant un sens historique pour le sujet. À ce niveau proprement narratif, la théorie de la construction de carrière de Savickas (Savickas \& Pouyaud, 2014 ; Savickas, 2011) permet d'étendre la métaphore. Dans cette approche théorique, l'identité narrative relie des micro-récits dans un macro-récit organisé autour d'une intrigue, des thèmes de vie et des personnages réalisant une histoire.

En résumé, l'identité narrative serait une réalisation temporaire et contextualisée d'un récit de soi, issu d'un montage dialogique des traces de l'expérience dans une reconstruction historique fidèle en vue d'un engagement dans une promesse de soi à tenir. L'identité construite au jour le jour est alors un point d'équilibre temporaire du bicycle permettant d'avancer.

\section{Francis Bacon et la construction de soi : analyse du dispositif narratif}

L'objet de cette partie est d'analyser comment l'œuvre de Bacon, trace de l'activité de construction de soi du peintre, construit, avec le spectateur, un dispositif dialogique qui interroge et mobilise les expériences d'altérité dans une perspective réflexive. 
l'image, la représentation, la forme, la métaphore et plus généralement la figura en latin : la chose façonnée, construite, modelée.

Cette Figure est souvent un visage avant d'être un corps. Le visage est construit pour Bacon autour de la bouche (un livre sur les maladies buccales a longtemps fasciné Bacon, plusieurs de ces peintures ne sont que des demi-visages où seule la bouche criante apparaît). Le regard est au contraire assez peu présent. La figure ne regarde jamais le spectateur. Elle observe plutôt quelque chose d'absent, hors cadre. Cette absence de regards croisés exclut le spectateur d'une communication possible pour mettre en place un dispositif d'auto-voyeurisme. La figure représentée est malléable, mobile, en perpétuelle métamorphose, insaisissable, et perçue comme un reflet viscéral de soi. Devant les tableaux de Bacon, le spectateur s'entr'aperçoit comme fuyant dans un miroir, avec le risque de se perdre de vue. Le spectateur ne peut qu'être voyeur de lui-même, le regard tout entier pris dans la peur de se perdre, n'ayant plus pour seule expression possible que le cri. La bouche est en ce sens la partie de la figure la plus importante, car elle exprime le sujet. Elle est pour Deleuze l'expression des organes internes, l'ouverture vers les profondeurs de la chair et la possibilité d'une évasion. Cette évasion, dans le refus de Bacon de laisser l'observateur construire du sens narratif, ne prend pas la forme d'une histoire, mais de celui, dans le cri, d'une énigme qui se vide: "Le cri de Bacon, c'est l'opération par laquelle le corps tout entier s'échappe par la bouche» (Deleuze, 2002, p. 24). L'énigme du corps qui s'évade est présente au-delà des portraits dans toutes les peintures; elle incite à voir le soi en dehors de la peinture d'où tous les repères narratifs sont supprimés. Pas de liens évidents entre les sujets, peu de décors, mais des image-énigmes que le spectateur ne doit pas chercher à reconstituer. Il faut plutôt les vivre et les fuir.

31 Si aucune figure n'est narrative, si le sujet est fuyant, qu'est-ce qui dans l'œuvre de Bacon constitue un soi consolidé? Dans la métaphore proposée plus haut, les sensations et marqueurs identitaires issus des émotions sont les supports d'un travail de montage du sujet par le langage. C'est par la force émotive, mais aussi par la succession, la répétition de ces marqueurs que vont s'élaborer des produits de montage identitaire pertinents. Les intérêts professionnels par exemple peuvent être vus comme ces produits montés à partir d'expériences particulièrement intenses dans un milieu ou auprès d'un professionnel, mais aussi les produits sédimentés d'un ensemble d'habitudes ou d'expériences quotidiennes organisées pour faire sens.

Ce n'est donc pas dans la peinture ou ses sujets qu'il faut chercher le sens chez Bacon, mais, comme le souligne Kundera, dans la répétition, répétition des sensations, des sujets et des figures comme autant de variations d'un même thème. Selon Kundera, ce qu'il y a de nouveau chez Bacon, c'est «le principe des variations. Husserl a expliqué l'importance des variations pour la recherche de l'essence d'un phénomène. [...] Les variations diffèrent l'une de l'autre, mais gardent en même temps un quelque chose qui leur est commun, c'est "ce trésor, cette pépite d'or, ce diamant caché", à savoir l'essence recherchée d'un thème, ou, dans le cas de Bacon, le "moi" d'un visage [...]. Je regarde les portraits de Bacon et je suis étonné que, malgré leur "distorsion", ils ressemblent tous à leur modèle [...] quoique "en distorsion" ces portraits sont fidèles " (Kundera, 1996, p. 11).

33 L'œuvre de Bacon est construite sur la variation des mêmes sujets; lutteurs, crucifixion, pape, tête, caméra, trois modèles et amis privilégiés: Georges Dyer et Lucian Freud, Isabelle Rawsthorne, et lui-même... Les sujets sont repris et repeints 
parfois avec des intervalles de plusieurs années comme c'est le cas par exemple du tableau Peinture fait en 1946 et "refait » en 1975. "L'acte pictural (est) une sorte de rappel.» (Deleuze, 2002, p. 68). Un peintre pour Bacon «s'ingénie à enfoncer avec obstination le même clou » (Bacon, 2012, p. 61), à « exprimer un sujet, qui est toujours le même et que l'on ne peut pas changer, mais en trouvant des formes à chaque fois nouvelles " (Bacon, 2012, p. 64). L'identité de ces figures, le sens de leur existence, la fidélité du portrait est une invention narrative qui n'apparaît pas dans les représentations isolées des tableaux qui ne restent que des potentialités narratives. Les personnages sont seuls dans des pièces, ils ignorent qu'ils sont observés, ils sont dans leur intimité la plus crue, seuls avec la violence de leur chair. « Sans doute peut-on dire que le tableau est la possibilité de toutes ces hypothèses ou narrations en même temps » (Deleuze, 2002, p. 69), mais pour en saisir le sens, pour dégager l'identité et sa construction, il faut reconstituer l'histoire de leur répétition picturale.

\section{Francis Bacon et la construction de l'identité}

Chaque peinture de Bacon illustre le processus de construction momentané d'une identité en mouvement qui tente désespérément de s'adapter. Chaque peinture met à nu le sujet dans sa confrontation à des altérités. Chez Bacon, l'activité de création est conditionnée par le lieu, l'atelier. Bacon dira qu'il ne peut travailler que dans cet atelier londonien où plusieurs photographies le montrent au milieu d'une montagne de papiers, les murs barbouillés et transformés en palettes géantes. Sa méthode consiste à entasser des "traces" de vie multiples, principalement des photos, coupures de journaux, traces de "sensations", de souvenirs vécus au hasard de l'existence. Ces traces finissent par former un amoncellement de matière qui envahit l'atelier comme un «tas de compost». La décomposition de la matière dans l'atelier offre à Bacon le substrat d'une création. Un document vient un jour à la surface, est saisi puis disparaît... Là encore, le hasard est orchestré en un mode de création propice à la variation, au recyclage perpétuel, aux métamorphoses. Le document (souvent une photographie) n'a que peu de valeur pour Bacon, car c'est une trace figée, au contraire de la peinture qui figure le mouvement de l'être (les photographies qu'affectionne Bacon sont celles qui évoquent le mouvement : photo d'identités en série prises dans un photomaton, les clichés de Muybridge sur la décomposition du mouvement). Les tableaux naissent des souvenirs, de sensations que le peintre cherche à restituer dans une "réalité " dynamique par la peinture. "La vie passe devant vous et vous la regardez, et voilà. On est tout le temps assailli par des images. Bien sûr, il y en a très peu qui restent, qui sont déterminantes, mais certaines ont un effet considérable. [...]. Il $\mathrm{y}$ a un effet de changement permanent qui se produit en moi. Certaines images, et peut être même tout ce que je vois, peuvent modifier imperceptiblement tout le reste " (Bacon, 2012, pp. 120-121). Ces images auxquelles Bacon s'attache sont pour Deleuze des " clichés ». Les clichés sont les substrats des narrations, «ce sont des images flottantes [...] qui circulent dans le monde extérieur, mais aussi qui pénètrent chacun et constituent son monde intérieur, si bien que chacun ne possède en soi que des clichés psychiques par lesquels il pense et il sent, étant lui-même un cliché parmi les autres dans le monde qui l'entoure » (Deleuze, 1983, p. 281).

Ce qui cimente la narration, ce "sont les clichés courants d'une époque ou d'un moment, slogans sonores et visuels [...] monologue intérieur d'un tiers quelconque, qui 
n'est pas identifié parmi les personnages" (Deleuze, 1983, p. 281). Pour Deleuze, la condition de la création passe par la destruction des clichés. Le créateur doit faire face au vertige de la destruction du monde pour détruire le cliché et produire une nouvelle figure inédite. Cette activité créatrice est de l'ordre d'un montage (au sens cinématographique), une activité de collage des sensations guidée par un «diagramme». "Le diagramme ne fonctionne jamais pour représenter un monde préexistant, il produit un nouveau type de réalité, un nouveau modèle de vérité. Il fait l'histoire en défaisant les réalités et les significations précédentes. [...] Il double l'histoire avec un devenir» (Deleuze, 1986, p. 43). Selon cette analyse, l'activité de peintre joue pour Bacon le rôle d'un langage intérieur et extérieur, à fonction diagrammatique, permettant un montage de ses sensations dans une réalisation renouvelée de soi, dont la figure centrale serait le triptyque.

\section{Francis Bacon et le langage du triptyque}

Le triptyque est de manière inattendue une des formes privilégiées par Bacon. Pour son projet non narratif, c'est une difficulté supplémentaire, car "il faut qu'il y ait un rapport entre les parties séparées, mais ce rapport ne doit être ni logique ni narratif. Le triptyque n'implique aucune progression, et ne raconte aucune histoire [...] Dès qu'il y a plusieurs figures [...] une histoire commence à s'élaborer. Et dès qu'une histoire s'élabore, l'ennui s'installe, l'histoire parle plus haut que la peinture » (Deleuze, 2002, pp. 68-69).

L'intérêt de Bacon pour le triptyque renvoie à une tentative de figuration athée de la condition humaine, ancrée dans le rapport à la chair plutôt que dans la spiritualité et la transcendance. Bacon s'inspire pour cela du cinéma, mais aussi du miroir à trois faces où le sujet peut se voir simultanément de face, de profil et de dos. La définition que donne Deleuze de la Figure reprend d'ailleurs cette caractéristique : la figure est un «signe qui, au lieu de renvoyer à son objet, en réfléchit un autre [...]; ou bien qui réfléchit son propre objet, mais en l'inversant [...]; ou bien qui réfléchit directement son objet » (Deleuze, 1983, p. 292).

Le triptyque est donc une forme de ce langage diagrammatique qui permet à Bacon le montage d'une identité narrative. Deleuze a décrit et analysé précisément ce langage dans La logique de la sensation (Deleuze, 2002). Pour lui, le triptyque est une peinture du rythme basé sur les relations entre les sensations. Ces relations rythmées par niveaux constituent la Figure dans sa dimension temporelle. La sensation est définie comme « ce qui se transmet directement, en évitant le détour ou l'ennui d'une histoire à raconter " (Deleuze, 2002, p. 41). «Le triptyque serait [ainsi] la distribution des trois rythmes de base " (Deleuze, 2002, p. 70), trois niveaux de sensations mis en relation rythmique pour construire une figure. Le premier rythme est la sensation simple qui est une "vibration ", le deuxième est la figure accouplée qui met en résonance deux sensations, et enfin le triptyque qui met en rapport les sensations entre elles selon trois « rythmes conjoints "; le rythme actif qui trouve son origine dans la sensation de descente ou de chute, "comme différence de niveau comprise dans la sensation" (Deleuze, 2002, p. 78). Ce rythme est celui de la croissance, du développement; le rythme passif qui constitue son contraire, une "variation décroissante, ou élimination " (Deleuze, 2002, p. 70) ; et enfin le rythme-témoin qui est une sensation horizontale basée sur les écarts entre les deux autres rythmes opposés (ascendant et descendant). Pour Deleuze, dans le 
triptyque, ce sont ces rythmes conjoints qui deviennent sensation à la manière d'une pièce de musique.

Deleuze déduit de cette analyse des « lois » de construction du triptyque chez Bacon, qui sont comme des grilles de lecture des sensations mises en relation, que l'on pourrait décrire aussi comme les traces d'un « diagramme de construction de soi » chez Bacon, une « logique de la sensation qui constitue la peinture» (Deleuze, 2002, p. 79) :

Mais une autre lecture du triptyque complémentaire de celle de Deleuze peut se faire en relation avec les propositions de Ricœur $(1988,1992)$ sur l'identité narrative comme " produit» d'un triple rapport à l'altérité. Quelles sensations provoque la première rencontre avec une peinture de Bacon? Certainement le plus souvent un malaise, une sensation de se retrouver face à l'homme dans son universalité, une sensation de confrontation avec une version de soi-même mise à nue, décomposée, désossée, et piégée dans un espace mental et physique indéfini, à la fois clos et temporellement infini. Ces tableaux par leur absence de sens et leur dimension instinctive provoquent toujours un sentiment de mystère. Le sentiment qu'il y a quelque chose sur soi-même caché derrière la figure, que certains vont aimer questionner, et que d'autres vont rejeter par peur ou dégoût du projet même de la découverte. Car ce mystère interroge notre propre rapport au corps, notre rapport à autrui, et notre rapport à nous-mêmes.

41 Si nous sommes si mal à l'aise face à ces tableaux, et en même temps si touchés au plus profond de nous-mêmes, c'est que nous nous y voyons comme dans un miroir, nous nous y voyons nous-mêmes comme un autre. Le triptyque chez Bacon est un dispositif sensationnel de confrontation à nous-mêmes, un moyen de faire résonner l'identité du spectateur en lui attribuant une place active dans la construction de la figure.

Il faut voir le triptyque comme le miroir à trois faces dans lequel le spectateur se confronte instinctivement aux trois expériences de l'altérité (être@corps, @autrui et @conscience), et construit dans le dialogue engagé une narration sur soi. Le triptyque est alors un dispositif de montage identitaire dans lequel se reflète une figure (au sens de Deleuze) de soi. Il offre la possibilité d'une « réflexion " pilotée par le langage et le langage intérieur et provoquée par la sensation. Dans cette rencontre active avec la peinture, la double réflexivité décrite par Guichard (2004) est bien présente et joue son rôle de moteur d'un développement de soi. La réflexivité duelle qui vise la stabilisation est en jeu dans l'identification "forcée » au sujet représenté - nous avons vu que cette contrainte relationnelle à la peinture découle directement de la méthode de création chez Bacon qui recherche l'accès direct au système nerveux. La réflexivité trinitaire vise la remise en question de soi. Elle est en jeu dans le rejet que provoque la peinture de Bacon, et dans une tentative du spectateur d'éliminer, de mettre à distance la figure choquante de lui-même qui lui est reflétée. Ainsi la violence, la barbarie permanente dans l'œuvre de Bacon n'est pas un exercice de style, mais une condition même d'un dispositif de révélation. C'est cette violence qui provoque le conflit du spectateur avec lui-même et permet la mise en œuvre d'un cri, et d'un dépassement. En ce sens, la double réflexivité n'est pas très éloignée du principe dynamique proposé par Deleuze entre rythme actif, passif et témoin. Le rythme témoin se trouvant peut être chez le spectateur lui-même, qui dans cet écart entre actif et passif, entre croissance et décroissance, entre amplification et élimination, entre stabilisation et remise en question, régule narrativement une temporalité reliant fidélité (au passé) et promesse (de l'avenir). 
Le triptyque Trois personnages dans une pièce (1964) en est une illustration. Dès la première rencontre, le spectateur est mis dans une relation identificatoire avec l'élément central du tableau. Ici au centre est présenté de face un personnage (qui est en fait un portrait de Bacon), mis en avant comme sur un piédestal, un fauteuil ou un trône. Autour vient ensuite pour le spectateur le sentiment d'une variation de ce personnage central. À gauche une vision de dos d'un sujet sur une cuvette de W.-C., il s'agit d'un portrait de Georges Dyer, à droite une vision de face d'un personnage cette fois-ci assis sur un simple siège, un portrait de Lucian Freud. Sur ce triptyque, Bacon a mis en confrontation, en relation sensorielle trois personnages (ami, amant) qui sont à la fois des personnages différents et des alter ego reliés entre eux par leur nudité. Les trois images sont ainsi des variations d'un même sujet, non pas seulement trois personnages dans une pièce, mais la sensation de « soi-même assis » plusieurs fois : une fois sur un trône, une autre sur des W.-C. (un autre trône), et enfin sur une chaise. La bouche, élément de relation entre l'intérieur et l'extérieur du corps n'est pas présente ici, mais est remplacée par les W.-C. dont un tuyau s'échappe vers l'extérieur du triptyque, suggérant l'évasion du corps, ou son écoulement par une bouche d'évacuation en dehors du cadre. C'est ce tuyau qui est l'énigme centrale sensorielle du tableau, et la source du conflit dans lequel le spectateur peut chercher un développement. Ce tuyau a à la fois l'aspect instinctivement " provocant » d'une chair qui s'écoule en déjection, mais aussi celui d'un décalage visuel ouvrant un mystère sur soi. C'est ce tuyau qui renvoie le spectateur à son rapport à la chair, à autrui et à soimême, et qui le pousse dans une narration interne et externe. L'énigme, qui au départ est une sensation visuelle, instinctive d'un décalage, d'un dérangement, induit une réflexivité qui transforme le spectateur en acteur par la mise en œuvre d'une conscience de soi relative à ce qu'il voit et ressent. Visuellement, c'est le tuyau qui rompt l'organisation circulaire du triptyque. En créant une coupure dans l'ovale de la pièce, dans l'armature fermée du triptyque, Bacon introduit une brèche pour une troisième dimension extérieure à la peinture, comme un legs qu'il fait au spectateur : celui de la conscience, de la temporalité et de la narration sur soi offert comme un cadeau à qui veut le prendre. En créant cette coupure, il transforme l'ovale fermé en une potentialité, une spirale narrative pour le spectateur, si ce dernier cherche à résoudre le conflit sensoriel et identitaire qui se pose à lui (c'est-à-dire dans la métaphore du bicycle, si il fait un effort de « pédalage » réflexif).

\section{Portrait de George Dyer à Bicyclette (1966)}

Parmi les nombreux portraits réalisés par Bacon de son ami et compagnon Georges Dyer, l'un d'eux offre une image particulièrement forte de la métaphore du bicycle. On y voit le personnage de profil, le visage isolé dans un contour sombre, avec le corps tout chargé des muscles en mouvement. L'effort est visible pour maintenir un équilibre qui n'est pas tant celui du sujet sur le vélo, mais de celui du couple que forment le sujet et le vélo ensemble, posé comme sur une armature de cirque, prêt pour un numéro d'équilibriste. Sujet et vélo ne font qu'un dans une posture qui suggère ici encore une sortie du cadre. Deleuze décrit cette toile ainsi : « les Figures de Bacon sont souvent saisies dans le vif d'une étrange promenade »(Deleuze, 2002, p. 44), où « il y a l'immobilité au-delà du mouvement» (Deleuze, 2002, p. 45), comme les "voyages immobiles » que Fernando Pessoa poétisait. Pour aller plus loin, la mise en relation de cette image avec la métaphore du bicycle pourrait faire de ce personnage esthétique un 
"personnage conceptuel» de la construction de soi, au sens défini par Deleuze et Guattari (1990). «Les personnages conceptuels ne sont pas, pour les deux auteurs, des personnes de chair et d'os, mais des formes de la pensée qui explicitent une définition philosophique " (Leblanc, 2012, p. 21). Pour que le lecteur y voie la métaphore de la construction de soi, il faudrait suivre les spirales hors du cadre. Imaginons un triptyque «à la manière » de Bacon. À droite, Georges Dyer à bicyclette, expression sensible d'un personnage conceptuel, à gauche, la figure diagrammatique métaphorique du vélo comme construction de soi (la figure 1, outil psychologique de compréhension ou plan de montage), au centre: un espace vide à remplir, le prolongement pratique ou théorique que le lecteur voudra construire de la confrontation des deux.

\section{Conclusion}

Le projet de cet article était d'analyser l'œuvre de Francis Bacon comme une trace confirmatoire d'un modèle actuel de la construction de soi utilisé en psychologie de l'orientation. Le paradigme du Life design sert de support à une métaphore de la construction de soi, présentant l'identité comme un bicycle permettant de "se" conduire. Plusieurs approches complémentaires sont mobilisées pour constituer ce collage métaphorique et théorique qui s'articule principalement autour du concept d'identité narrative. L'œuvre de Bacon est alors analysée à partir de la manière dont elle est produite par le peintre pour explorer à la fois comment elle exprime ce dernier en tant que sujet, mais aussi comment elle met en œuvre concrètement le modèle de la construction de soi dans la relation sensorielle créée par l'observateur avec la peinture.

À travers cette analyse, c'est aussi plus globalement un questionnement sur les caractéristiques du sujet actuel et de ses mutations qui est posé. Bacon est profondément ancré dans le $\mathrm{xx}^{\mathrm{e}}$ siècle, mais cette métaphore suggère peut-être aussi autre chose du sujet actuel, ayant trait à la question du dépassement et de l'outillage. La subjectivité est avant tout l'affaire de l'homme dans sa capacité de conscience libre et autonome. Mais les évolutions du $\mathrm{xxI}^{\mathrm{e}}$ siècle posent plus loin la question du dépassement des individus dans leur propre conscience. Derrière la désynchronisation, la saturation du soi, il y a aussi peut être une incapacité des individus à faire face seuls à une dilution, une diffusion, un éparpillement, un écoulement des consciences dans des réseaux dématérialisés.

En ce sens, le cri peint par Bacon est peut-être aussi prémonitoire. Ce cri de terreur peut être un appel au secours du sujet face à une conscience qui ne peut plus être contenue dans un corps, et qui s'échappe, se désagrège et s'écoule sans entrave.

La métaphore du bicycle pose la question de la confrontation à cette limite, à la fuite des consciences et au besoin d'outillage pour se développer. Le vélo est apparu à l'aube $\mathrm{du} \mathrm{xx}^{\mathrm{e}}$ siècle comme un outil de libération, comme une nouvelle capacité de déplacement autonome, une démultiplication du pouvoir d'action. Le sujet actuel a-t-il besoin d'un nouveau vélo, un nouvel outil de déplacement, non plus physique, mais psychique, une extension de soi qui lui facilite la tâche de démultiplication rendue nécessaire par les évolutions sociétales? Les nouvelles technologies et avancées médicales transforment de jour en jour nos corps en cyborgs connectés. Elles transforment notre rapport à nous-mêmes et aux autres, elles construisent au jour le jour une dépendance de plus en plus forte à l'artificialité. Qui serons-nous quand notre identité dépendra uniquement d'un réseau d'information que personne ne maîtrisera si 
ce n'est le réseau lui-même? Comment nous définirons-nous quand la majeure partie de notre expérience corporelle sera déplacée ailleurs et vécue à distance ? C'est aussi à cette dématérialisation de l'identité que Bacon nous pousse à réfléchir en nous ramenant au corps.

\section{BIBLIOGRAPHIE}

Adda, E. (éd.). (1996). Bacon : Portraits et Autoportraits. Paris : Archambaud, Les Belles Lettres. Bacon, F. (2012). Francis Bacon, Entretiens avec Michel Archimbaud (traduit par C. Le Gallic). Paris : Gallimard.

Bauman, Z. (2007). Liquid Times: Living in an Age of Uncertainty. Cambridge: Polity Press.

Deleuze, G. (1983). Cinéma 1. L'image-mouvement. Paris : Éditions de Minuit.

Deleuze, G. (1986). Foucault. Paris : Éditions de Minuit.

Deleuze, G. (2002). Francis Bacon : La logique de la sensation. Paris : Seuil.

Deleuze, G., \& Guattari, F. (1990). Qu'est-ce que la philosophie ? Paris : Éditions de Minuit.

Ehrenberg, A. (1995). L'individu incertain. Paris : Calmann-Lévy.

Fischbach, F. (2011). La privation du monde. Paris : Vrin.

Fruteau de Laclos, F. (2007). đuvre, fonction et société dans la « psychologie historique » d'Ignace Meyerson. Revue d'histoire des sciences humaines, 17, 119-136.

Gergen, K. (2009). Le soi saturé. Bruxelles : Satas.

Guichard, J. (2000). Cadres et formes identitaires vicariantes et pratiques en orientation. L'Orientation scolaire et professionnelle, 29(1), 181-212.

Guichard, J. (2004). Se faire soi. L'Orientation scolaire et professionnelle, 33(4), 499-533.

Guichard, J. (2009). Self-Constructing. Journal of Vocational Behavior, 75(3), 251-258.

Guichard, J., \& Pouyaud, J. (2014). Processes of Identity Construction in Liquid Modernity: Actions, Emotions, Identifications and Interpretations. In R. A. Young, L. Valach, \& J. F. Domene (eds.), Counseling and Action: Toward Life-Enhancing Work, Relationships, and Identity. New York: Springer Science.

Harrisson, C., \& Wood, P. (1997). Art en théorie 1900-1990. Paris : Hazan.

Hartung, P. J. (2013). Career as Story: Making the Narrative Turn. In W. B. Walsh, M. L. Savikas, \& P. J. Hartung (eds.), Handbook of Vocational Psychology: Theory, Research, and Practice (4rd ed.). New York: Routledge.

Jacques, F. (1982). Différence et subjectivité. Paris : Aubier.

Kundera, M. (1996). Le geste brutal du peintre. In E. Adda (éd.), Bacon. Paris : Archambaud, Les Belles Lettres.

Leblanc, G. (2012). Courir : Méditation physique. Paris : Flammarion. 
McMahon, M., \& Patton, W. (eds.) (2005). Career Counselling: Constructivist Approaches. New York: Routledge.

McMahon, M., \& Watson, M. (eds.) (2011). Career Counseling and Constructivism: Elaboration of Constructs. New York: Nova Science Publishers Inc.

Meyerson, I. (1995). Les fonctions psychologiques et les œuvres. Paris : Albin Michel.

Pouyaud, J. (2014). Vocational Trajectories and Peoples' Multiple Identities: A Life Design. In L. Nota \& J. Rossier (eds.), Handbook of the Life Design Paradigm: From Practice to Theory, from Theory to Practice. Toronto: Hogrefe \& Huber Publishers.

Ricœur, P. (1988). L'identité narrative. Esprit, 7(8), 295-314.

Ricœur, P. (1992). Soi-même comme un autre. Paris : Seuil.

Rosa, H. (2012). Aliénation et accélération. Paris : La Découverte.

Savickas, M. L. (2011). Career Counseling. Washington, DC US: American Psychological Association. Savickas, M. L., Nota, L., Duarte, M. E., Guichard, J., Soresi, S., Van Esbroeck, R., \& Bigeon, C. (2010). Construire sa vie (Life designing) : un paradigme pour l'orientation au $21^{\mathrm{e}}$ siècle. L'Orientation scolaire et professionnelle, 39(1), 1-20.

Savickas, M. L., \& Pouyaud, J. (2014). Concevoir et construire sa vie : un modèle général pour l'accompagnement en orientation au XxI ${ }^{\mathrm{e}}$ siècle. Psychologie française.

Sloterdijk, P. (2000). La mobilisation infinie. Paris : Bourgois.

Sylvester, D. (2013). Francis Bacon : Entretiens (traduit par M. Leiris, F. Gaillard, M. Peppiatt \& P. Sylvester). Paris : Flammarion. ( $1^{\text {re }}$ édition : 1976).

Vygotski, L. S. (1985). La méthode instrumentale en psychologie. In B. Schneuwly \& J.-P. Bronckart (éd.), Vygotsky aujourd'hui (pp. 39-47). Neuchâtel : Delachaux et Niestlé.

Vygotski, L. S. (1997). Pensée et langage. Paris : La Dispute.

Young, R. A., \& Valach, L. (2004). The Construction of Career Through Goal-Directed Action. Journal of Vocational Behavior, 64(3), 499-514.

Young, R. A., \& Valach, L. (2006). La notion de projet en psychologie de l'orientation. L'Orientation scolaire et professionnelle, 35(4), 495-509.

\section{NOTES}

1. . Vygotski cite d'ailleurs Francis Bacon le philosophe en épigraphe de Pensée et langage comme source d'inspiration centrale: «Nec manus, nisi intellectus, sibi permissus, multam valent instrumentis et auxilibus res perficitur » [la main et l'intelligence humaines, privées des outils nécessaires et des auxiliaires, restent assez impuissantes]; inversement, ce qui renforce leur puissance, ce sont les outils et les auxiliaires offerts par la culture. 


\section{RÉSUMÉS}

Francis Bacon est un peintre dont l'œuvre entière est emblématique du $\mathrm{xx}^{\mathrm{e}}$ siècle et du tournant vers la postmodernité. L'article prend comme point d'appui le travail du peintre pour aborder les questions de la construction de soi et du Life Design, au centre des modèles actuels utilisés en psychologie de l'orientation. Les modèles principaux de ces paradigmes constructivistes, dont la notion d'identité est un pilier, sont résumés par une métaphore qui rapproche l'identité de l'image du vélo permettant de "se conduire ", illustrant ainsi les enjeux de la construction de soi. Ces modèles s'articulent autour du concept d'identité narrative développé par Ricoeur. L'article présente comment l'œuvre de Bacon, analysée comme trace de l'activité de construction de soi, illustre ces modèles.

Francis Bacon is certainly one of the most representative painter of the $\mathrm{XX}^{\text {th }}$ century and of the turning point into postmodernity. The article starts from an analysis of his work to illustrate current models of self construction and life design in the field of career counseling. The article proposes to follow the metaphor of the Bicycle (Be-cycle) to blend complementary theoretical models of self construction, organized around Ricoeur's concept of narrative identity. It describes how Bacon's work can be analysed as a trace of his own self construction activity, and therefore as a figure for our own self construction.

\section{INDEX}

Mots-clés : Life design, construction de soi, postmodernisme, identité, identité narrative, processus créatif

Keywords : Life design, Self construction, Postmodernism, Identity, Narrative Identity, creation process

\section{AUTEUR}

\section{JACQUES POUYAUD}

est Maître de conférences, Université de Bordeaux. Thèmes de recherche : activité de conseil, construction de l'identité, transitions. Contact : Université de Bordeaux, 3 Ter Place de la Victoire 33000 Bordeaux. Courriel : jacques.pouyaud@u-bordeaux.fr 